\title{
Fournier's Gangrene in a Patient Receiving Treatment for Idiopathic Thrombocytopenic Purpura
}

\author{
Junichiro Yuda $^{1}$, Riko Honma ${ }^{1}$, Tomoyasu Yahagi ${ }^{2}$ and Eijiro Omoto ${ }^{1}$
}

\begin{abstract}
We report the case of a 68-year-old man who was diagnosed with Fournier's gangrene (FG), which developed during immunosuppresive treatment for idiopathic thrombocytopenic purpura (ITP). The patient was administered steroids for ITP but on the 36th day, he developed FG and septic shock. We initiated antibiotic treatment and drained a periproctal abscess immediately. On day 53, extensive drainage to progressive FG and a splenectomy was performed, following which both FG and thrombocytopenia improved. This is the first case of FG has developing in a ITP patient. It appears that high-dose immunoglobulin therapy and splenectomy should be considered earlier especially for a patient complicated with FG.
\end{abstract}

Key words: idiopathic thrombocytopenic purpura, Fournier's gangrene, septic shock

(Intern Med 50: 2015-2019, 2011)

(DOI: 10.2169/internalmedicine.50.5323)

\section{Introduction}

In 1883, Fournier first described Fournier's gangrene (FG) as a life threatening soft tissue gangrene of unkown origin that is found around the perineum up to the anus. This condition is now recognized as a rapidly progressive necrotizing fasciitis (NF), that spreads to the gluteal muscles, and to the scrotum and penis in males. Penetration of the gastrointestinal or urethral mucosa can cause FG, which is induced by a mixture of aerobic and anaerobic organisms, also involving enteric organisms. Despite the advances in modern intensive care techniques and medical treatment, FG is associated with a high level of morbidity and mortality. Indeed, an aggressive approach must be adopted to successfully combat this disease, involving immediate surgical drainage and the administration of broad-spectrum antibiotics. Here we report the case of patient that developed FG due to Klebsiella pneumoniae while he was being treated for idiopathic thrombocytopenic purpura.

\section{Case Report}

A 68-year-old man was admitted to our hospital, com- plaining of fatigue, nasal bleeding and petechia of the lower extremities. In his past history, it was noteworthy that he had undergone surgery for an anal fistula in his twenties and that he had been diagnosed with diabetes mellitus in his sixties.

\section{Findings on admission}

The patient was $162 \mathrm{~cm}$ tall and weighed $92 \mathrm{~kg}$ (giving a BMI of $35 \mathrm{~kg} / \mathrm{m}^{2}$ ). His blood pressure was $130 / 74 \mathrm{mmHg}$, and his body temperature was $36.3^{\circ} \mathrm{C}$. No murmur and no rales were heard, and there were no abnormal findings in the abdominal region. Petechia was evident bilaterally in the lower extremities.

\section{Diagnosis}

Clinical laboratory tests showed that the patient's platelet counts was $2,000 / \mu \mathrm{L}$ and his platelet-associated immunoglobulin $\mathrm{G}(\mathrm{PAIgG})$ titer had risen to $1,650 \mathrm{ng} / 10^{7}$ cells (Table 1). Examination of the bone marrow showed that there was hyperplasia of megakaryocytes but no dysplasia of hematopoietic cells. After ruling out other diseases that could induce thrombocytopenia, including collagen disease, autoimmune disease and disseminated coagulating syndrome, a diagnosis of ITP was made. The patient visited our hospital

${ }^{1}$ Department of Hematology, Yamagata Prefectural Central Hospital, Japan and ${ }^{2}$ Department of Cardiology, Yamagata Prefectural Central Hospital, Japan

Received for publication February 14, 2011; Accepted for publication May 23, 2011

Correspondence to Dr. Eijiro Omoto, eomoto@ypch.gr.jp 
Table 1. Laboratory Findings on Admission

\begin{tabular}{|c|c|c|c|c|}
\hline $\mathrm{RBC}$ & & $467 \times 10^{4} / \mu \mathrm{L}$ & AST & $22 \mathrm{IU} / \mathrm{L}$ \\
\hline HBG & & $14.6 \mathrm{~g} / \mathrm{dL}$ & ALT & $23 \mathrm{IU} / \mathrm{L}$ \\
\hline $\mathrm{HCT}$ & & $42.4 \%$ & LDH & $308 \mathrm{IU} / \mathrm{L}$ \\
\hline MCV & & $90.8 \mathrm{fL}$ & T-Bil & $0.3 \mathrm{mg} / \mathrm{dL}$ \\
\hline $\mathrm{MCH}$ & & $31.3 \mathrm{pg}$ & BUN & $14.5 \mathrm{mg} / \mathrm{dL}$ \\
\hline $\mathrm{MCHC}$ & & $34.4 \%$ & CRE & $0.99 \mathrm{mg} / \mathrm{dL}$ \\
\hline PLT & & $2000 / \mu \mathrm{L}$ & CRP & $0.099 \mathrm{mg} / \mathrm{dL}$ \\
\hline RET & & $4.53 \times 10^{4} / \mu \mathrm{L}$ & Fibrinogen & 308 mg/dL \\
\hline \multirow[t]{7}{*}{ WBC } & & $5370 / \mu \mathrm{L}$ & FDP & $2.6 \mu \mathrm{g} / \mathrm{dL}$ \\
\hline & Band & $1.0 \%$ & D-Dimer & $0.8 \mu \mathrm{g} / \mathrm{dL}$ \\
\hline & Seg & $47.0 \%$ & PAlgG & $1650 \mathrm{ng} / 10^{7}$ cells \\
\hline & Eosino & $3.0 \%$ & H.Pylori-Ab (urine) & negative \\
\hline & Baso & $1.0 \%$ & H.Pylori-Ag (stool) & negative \\
\hline & Mono & $8.0 \%$ & & \\
\hline & Lymph & $40.0 \%$ & & \\
\hline
\end{tabular}

within a week of the onset and had no previous therapy. Because the patient had no evidence of Helicobacter pylori infection, we decided to commence steroid therapy first.

\section{Clinical course (see Fig. 1)}

On day 8 after admission, we began to administer $1 \mathrm{mg} /$ $\mathrm{kg}$ *day of prednisolone (PSL), however, only a small increase in the platelet count to $20,000 / \mu \mathrm{L}$ was observed. For this reason, we increased the dose of PSL to $2 \mathrm{mg} / \mathrm{kg}$ *day on the 22nd day. His blood sugar became higher as an adverse event of steroid. Steroid therapy exaggerated diabetes but his blood sugar levels were relatively well controlled within $100-200 \mathrm{mg} / \mathrm{dL}$. On day 31 the patient developed painful bleeding from the anus. The patient presented on day 35 with urinary retention and therefore an urethral catheter was inserted. On the following day, the patient developed a fever with septic shock. Laboratory results showed severe inflammation. A CT scan revealed emphysematous gangrene of the perineum and pneumoperitoneum (Fig. 2). These results confirmed a diagnosis of Fournier's gangrene (FG) due to anal fistula infection. At that time, FDP and D-Dimer levels were within nomal limits, so he was not in disseminated intravascular coagulation (DIC) status. The patient was treated with extensive fluid replacement, catecholamines and antibiotics (including doripenem hydrate and linezolid), as well as surgical drainage from under local anesthesia. Klebsiella pneumoniae was cultured from the patient's blood and necrotizing tissues. At the same time, cytomegalovirus (CMV) antigens were evident in 46/ 50,000 white blood cels (WBC) and hence, ganciclovir administration was initiated. We thought more aggressive surgical drainage, including a laparotomy, would be needed for the patient to recover from this status, but low platelet count was the obstacle to perfoming laparotomy. An attempt to increase platlets with high-dose immunoglobulin was in vain. On day 49 , the platelet count was still $17,000 / \mu \mathrm{L}$ under 1 $\mathrm{mg} / \mathrm{kg} / \mathrm{day}$ of PSL and all antibiotics were discontinued because the patient was recovering from the septic shock. On day 54, he experienced severe lower abdominal pain and again fell into septic shock status for a second time. A CT scan revealed that the abscess and gangrene had expanded. The FG was thought to be still progressing (Fig. 3). His spontaneous breathing stopped 4 hours after entering into shock, and he was intubated and mechanical ventilation was established in the intensive care unit (ICU). Immediately, we reinitiated treatment with several antibiotics and dopamine again. The patient presented renal dysfunction and endotoxic lipopolysaccharide was evident in the blood. We performed dialysis and polymixin-B direct hemoperfusion (PMX-DHP) therapy. Despite these treatment, his systolic blood pressure remained at around $50 \mathrm{mmHg}$ for 15 hours. We also initiated more aggressive surgical drainage, as well as anticoagulation therapy for DIC, which gradually improved his general condition. The FDP and D-Dimer levels were controlled within nomal limits. Although the patient's platelet counts were not sufficiently high, we performed surgical drainage, a splenectomy and an orchiectomy with platelet transfusion under general anesthesia on day 66. At the same time, we carried out a colostomy and a cystostomy. On day 73, a CT scan still revealed the presence of prostatic abscess and bilateral subcutaneous abscess in the abdomen, although the necrotizing lesion had diminished. After extubation, consciousness level of the patient was still not clear and he suffered a dysarthria. On day 78, MRI examination revealed a high intensity area in the bilateral basal ganglia due to hypoxic damage, which was associated with the prolong septic shock. On day 84, the patient's platelet count had risen to $727,000 / \mu \mathrm{L}$ and his general condition and level of consciousness had improved sufficiently that he was moved to a general ward from the ICU. The value of PAIgG was $96 \mathrm{ng} /$ $10^{7}$ cells on day 109.

\section{Discussion}

FG is an uncommon necrotizing fasciitis (NF) of genital and periproctal lesions that leads to thrombosis of small subcutaneous vessels resulting in the development of gangrene in the overlying skin. The Fournier's Gangrene Severity Index (FGSI) score is a numeric score obtained from a combination of admission physiologic parameters, including temperature, heart and respiration rates, sodium, potassium, creatinine, WBC counts, hematocrit, and sodium bicarbonate. It has been proposed that a FGSI score $>9$ indicates a $75 \%$ probability of mortality and score of $\leqq 9$ is associated with a $78 \%$ probability of survival (1). In the present patient, the FGSI score reached 12 and thus, the probability of mortality was thought to be high. There are seveal risk factors for NF, including immunosuppression, diabetes mellitus, obesity, malignancy, drug abuse and chronic kidney disease (2-7). In terms of hematological diseases, a case of NF with leukemia has also been reported (8), but there are no reports of NF associated with ITP. Our patient had a history of diabetes mellitus and he had received high doses of PSL. His blood sugar on admission seemed to be well controlled, 


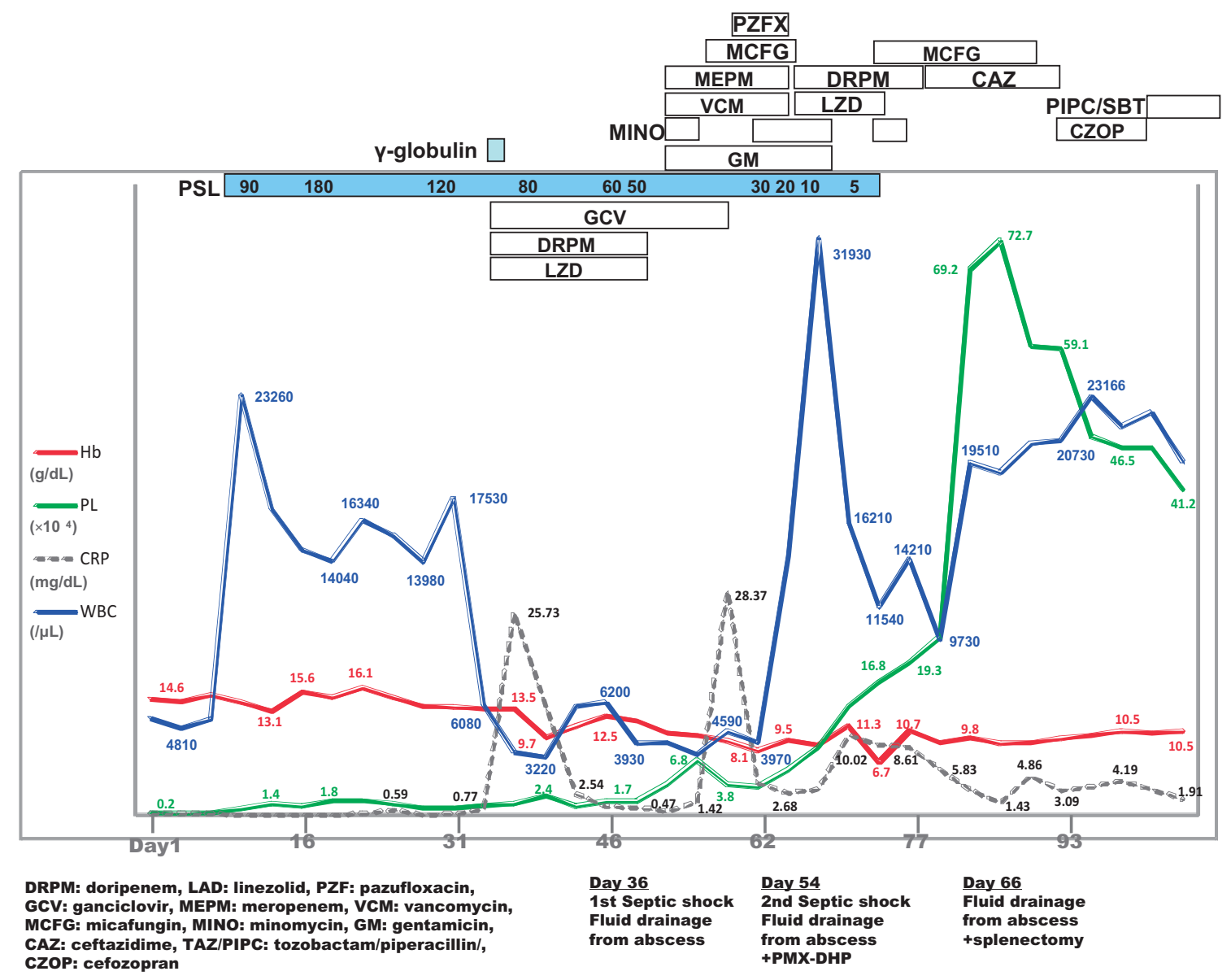

Figure 1. Clinical course.

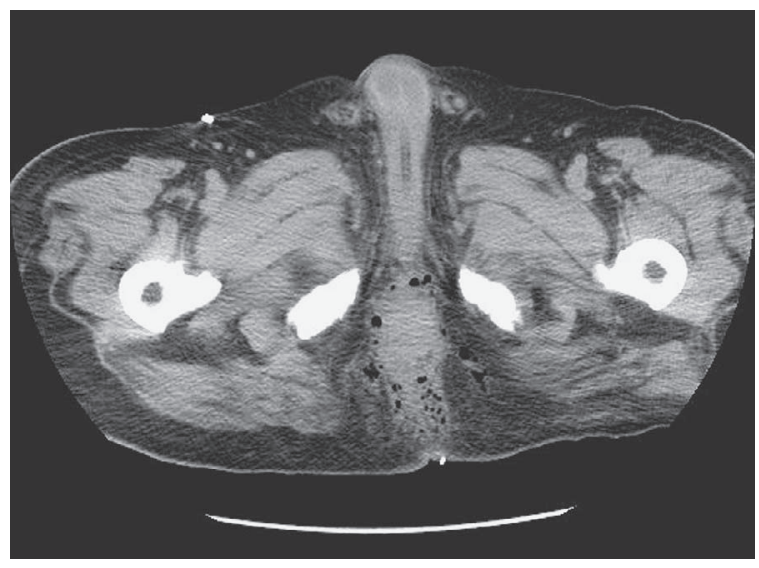

Day 36

CT scan revealed the emphysematous gangrene of peritoneum.

Figure 2. CT scan.

although it worsened, and his immune system was severely suppressed by the administration of the steroid, leading to the development of FG and CMV infection. CMV infection is also thought to be an opportunistic infection of individuals under severe immunosuppressive stress. Indeed, a FG patient has been identified previously who was administered steroids to treat lupus nephritis (9). About $50 \%$ of FG patients have a history of skin injury and although this was

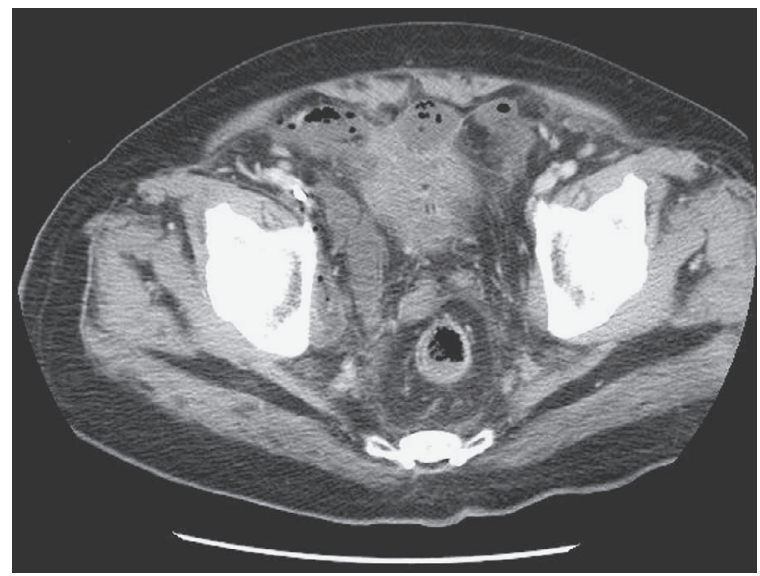

Day 54

CT scan revealed that the abscess and gangrene were expanding.

Figure 3. CT scan.

not the case with this patient, he had developed an anal fistula about 50 years previously, which seems to have been the trigger for developing FG. NF is categorized as type 1 or 2, and other micicrobiological etiologies are evaluated on the basis of microbiological cultures (10-14). Type 1 NF involves a polymicrobial infection by aerobic and anaerobic bacteria in people who are immunocompromised or who suffer a chronic disease like diabetes mellitus. Type $2 \mathrm{NF}$ 
involves group A Streptococcus (GAS) infection, with or without a concomitant streptococcal infection, and it can occur in any age group even in the absence of any complicated medical illness. Indeed, only a single micro-organism is found in the blood of $27 \%$ of NF patients, whereas multiple micro-organisms are identified in the blood of $2 \%$ of $\mathrm{NF}$ patients and no micro-organisms are found in $71 \%$ of NF cases (15). Streptococcus spp., S. aureus, V. vulnificus, A. hydrophila, Enterobacteriaceae (Escherichia coli, Pseudomonas spp., and Klebsiella spp.) and anaerobic streptococcus have all been detected as monomicrobial infections. However, in the present patient, only Klebsiella pneumoniae was initially detected in the blood, while both Klebsiella pneumoniae and Enterococcus faecalis were subsequently cultured from the necrotizing tissue. When we identified this polymicrobial infection, combined antibiotics were administered to combat both gram positive cocci and gram negative rods. However, the patient developed both ventilatorassociated pneumonia and hospital-acquired pneumonia, and Stenotrophomonas maltophilia, Pseudomonas putida, Sphingomonas paucimobilis, Candida albicans and Candida parapsilosis were detected in his sputum. We changed antibiotics several times according to the result of cultures and his pneumonia status. PMX-DHP has been reported to be effective in treating abdominal sepsis (16), and when performed, it produced a good response to the second septic shock in the present patient. After the first septic shock, the infection was gradually controlled and the patient's platelet count temporarily rose to $68,000 / \mu \mathrm{L}$. Accordingly, it seemed that his platelet counts were influenced by the infection. DIC might have influenced the counts, but D-dimer values were not correlated to platelet counts. If the administration of antibiotics was continued after the first septic shock, the second septic shock might be evitable. In this case, early discontinuation of antimicrobial treatment seems to be related to recurrence of $\mathrm{FG}$, suggesting the requirement of continuous aggressive antimicrobial therapy until the aggressive surgical drainage is performed. In adults, hepatitis $\mathrm{C}$ virus, human immunodeficiency virus and Helicobacter pylori are the most prevalent infections known to cause thrombocytopenia (17). Indeed, there is no evidence that Klebsiella pneumoniae infections induce thrombocytopenia. We consider that the poor control of infection might stimulate a patient's immune system, including the self-reactive immune system associated with ITP. Thus, if this patient's periproctal lesion was already infected, this could have contributed to the onset of ITP. Furthermore the immunosuppression induced by the steroids administered would have worsened this infection, introducing the patient into a pathogenic vicious circle. Accordingly, infections in ITP patients should be closely monitored during immunosuppressive therapy. Significantly, 9\% of FG patients have been reported to be medically immunosuppressed (18).

Platelet membrane glycoprotein IIb-IIIa (GP IIb/IIIa) and glycoprotein Ib-IX (GPIb/IX) are known as the major targets of the anti-platelet antibodies. Anti-GP IIb/IIIa and anti-GP
$\mathrm{Ib} / \mathrm{IX}$ antibodies should have been checked in this patient. The activation of autoreactive $\mathrm{T}$ and $\mathrm{B}$ cells to GP IIb/IIIa antibody production occurs primarily in the spleen in the majority of ITP patients. Enzyme-Linked ImmunosSpot (ELISPOT) assay enables detection and quantification of anti-GP IIb/IIIa antibody levels produced by B cells (19).

Although a splenectomy might have been the only effective treatment for ITP in this case, the thrombocytopenia detected made us hesitate before performing the operation. Patients that do not respond to glucocorticoid or intravenous immunoglobulin administration, and who therefore require splenectomy, are at greater risk of death from bleeding than those who do respond to these drugs. The rate of death from bleeding in patients with ITP that were subjected to a splenectomy by laparotomy was reported to be $0.2 \%$ (11/ 4955) (20). There does not appear to be a strong correlation between the effectiveness of splenectomy and previous intravenous immunoglobulin administration. Most adults with ITP respond to PSL within two weeks, with the majority responding within the first week. Only $18.7 \%$ of the patients had a sustained complete remission lasting more than six months after maintenance therapy was discontinued (21). Also, the effect of intravenous immunoglobulin was only transient. Because the patient was at high risk of developing FG, we should performed the splenectomy earlier after comfirming the ineffectiveness of intravenous immunoglobulin rather than administrating steroids for such a lengthy period.

In summary, when treating ITP patients, it appears to be important to first check carefully for any foci of infection. In ITP patients at high risk of FG, glucocorticoids should not be administered and when signs of FG are found, a splenectomy and aggressive surgical drainage (22) should be performed as soon as possible after initiating intravenous immunoglobulin therapy.

The authors state that they have no Conflict of Interest (COI).

\section{References}

1. Laor E, Palmer LS, Tolia BM, et al. Outcome prediction in patients with Fournier's gangrene. J Urol 154: 89-92, 1995.

2. Anaya DA, Dellinger EP. Necrotizing soft-tissue infection: diagnosis and management. Clin Infection Dis 44: 705, 2007.

3. Eliott DC, Kufera JA, Myers RA. Necrotizing soft tissue infections. Risk factors for mortality and strategies for management. Ann Surg 224: 672-683, 1996.

4. Kotrappa KS, Bansal RS, Amin NM. Necrotizing fasciitis. Am Fam Physician 53: 1691-1697, 1996.

5. Gonzalez MH. Necrotizing fasciitis and gangrene of the upper extremity. Hand Clin 14: 635-645, 1998.

6. Green RJ, Dafoe DC, Raffin TA. Necrotizing fasciitis. Chest 110: 219-229, 1996.

7. Chapnick EK, Abter EI. Necrotizing soft-tissue infections. Infect Dis Clin North Am 10: 835-855, 1996.

8. Lorenzen JM, Krauter J, Langer F, Haller H, Gwinner W. Necrotizing faciitis and acute kidney injury in a patient with acute myelogenous leukemia: Case presentation and review of the literature. Ann Hematol 90: 235-238, 2011.

9. Kohagura K, Sesoko S, Tozawa M, Iseki K, Tokuyama K, Fuki- 
yama K. A female case of Fournier's gangrene in a patient with lupus nephritis. Nippon Jinzo Gakkai Shi 40: 354-358, 1998 (in Japanese).

10. Bison AL, Stevens DL. Streptococcal infections of skin and soft tissues. N Engl J Med 334: 240-245, 1996.

11. Fontes RA Jr, Ogilvie CM, Miclau T. Necrotizing soft-tissue infections. J Am Acad Orthop Surge 8: 151-158, 2000.

12. Elliot D, Kufera JA, Myers RA. The microbiology of necrotizing soft tissue infections. Am J Surg 179: 361-366, 2000.

13. Shimizu T, Tokuda Y. Necrotizing fasciitis. Intern Med 49: 10511057,2010

14. Saenz EV, Magro MH, Ovalle V, et al. Experience in management of Fournier's gangrene. Tech Coloproctol 6: 5-13, 2002.

15. Hsiao CT, Weng HH, Yuan YD, Chen CT, Chen IC. Predictors of mortality in patients with necrotizing fasciitis. Qm J Emerg Med 26: 170-175, 2008.

16. Cruz DN, Antonelli M, Fumagalli R, et al. Early use of polymyxin B hemoperfusion in abdominal septic shock. JAMA 301: 2445-2452, 2009.

17. Stasi R, Willis F, Shannon MS, et al. Infectious causes of chronic immune thrombocytopenia. Hematol Oncol Clin N Am 23: $1275-$ 1297, 2009.

18. Tahmaz L, Erdemir F, Kibar Y, et al. Fournier's gangrene: report of thirty-three cases and a review of the literature. Int $\mathrm{J}$ Urol 7: 960-967, 2006.

19. Kuwana M, Okazaki Y, Kaburaki J, Kawakami Y, Ikeda Y. Spleen is a primary site for activation of platelet-reactive $\mathrm{T}$ and $\mathrm{B}$ Cells in patients with immune thrombocytopenic purpura. J Immunol 168: $3675-3682,2002$.

20. Kojouri K, Vesely SK, Terrell DR, George JN. Splenectomy for adult patients with idiopathic thrombocytopenic purpura: a systematic review to assess long-term platelet count responses, prediction of response, and surgical complication. Blood 104: 2623-2634, 2004.

21. Stasi R, Stipa E, Masi M, et al. Long-term observation of 208 adults with chronic idiopathic thrombocytopenic purpura. Am $\mathrm{J}$ Med 98: 436-442, 1995.

22. McHenry CR, Piotrowski JJ, Petrinic D, et al. Determinants of mortality for necrotizing soft-tissue infections. AnnSurg 221: 558$563,1995$.

(C) 2011 The Japanese Society of Internal Medicine http://www.naika.or.jp/imindex.html 